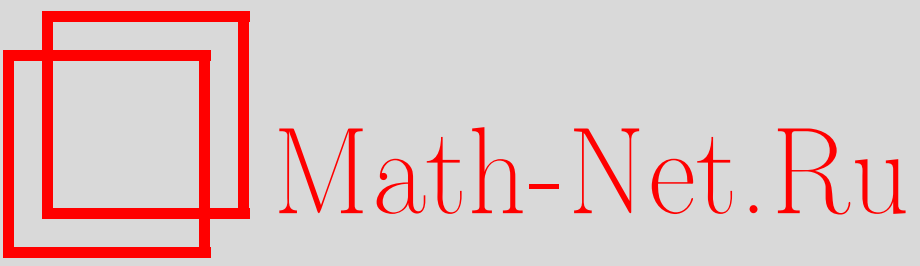

Я. М. Клебанов, И. Е. Адеянов, Распараллеливание решений задач деформирования при пластическом разупрочнении, Вестн. Сам. гос. техн. ун-та. Сер. Физ.-мат. науки, 2011, выпуск 2(), 89-100

DOI: https://doi.org/10.14498/vsgtu792

Использование Общероссийского математического портала Math-Net.Ru подразумевает, что вы прочитали и согласны с пользовательским соглашением

http: //www . mathnet.ru/rus/agreement

Параметры загрузки:

IP : 3.95 .254 .165

26 апреля 2023 г., $17: 00: 24$ 
УДК 519.681.5:517.958:539.3

\section{РАСПАРАЛЛЕЛИВАНИЕ РЕШЕНИЙ ЗАДАЧ ДЕФОРМИРОВАНИЯ ПРИ ПЛАСТИЧЕСКОМ РАЗУПРОЧНЕНИИ}

\section{Я. М. Клебанов, И.Е. Адеянов}

Самарский государственный технический университет, 443100, Самара, ул. Молодогвардейская, 244.

E-mails: jklebanov@mail.ru, adigorev@gmail.com

Рассматривается метод распараллеливания решений связанных краевых задач дебормирования и повреждённости материала при разупрочнении. Использовался принцип эквивалентности дебормаций в реальной и условно неповреждённой конструкииях. Предлагается итерационная процедура, особенностъю которой является то, что на каждой из итераций последователъно решается сначала краевая задача пластического деформирования, а затем - повреждённости. Подход $к$ распараллеливанию задач пластического разупрочнения основан на использовании концепции нелинейных обобщённых моделей конструкций и методе декомпозиции.

Ключевые слова: распараллеливание, конструкиия, обобщённая моделъ, декомпозиция, разупрочнение, краевая задача, нелокальная пластическая дебормация.

Введение. Сложность решения нелинейных задач деформирования конструкций является главной причиной того, что в настоящее время значительное внимание уделяется развитию численных методов и использованию вычислительной техники. Появление доступных параллельных ЭВМ вызвало повышение интереса к распараллеливанию решений краевых задач. Целью данной работы является развитие подхода к распараллеливанию нелинейных задач механики деформируемого твёрдого тела, предложенного в $[1,2]$. Этот подход основан на концепции нелинейных обобщённых моделей конструкций и методе декомпозиции. Моделирование на параллельных ЭВМ, использующее метод обобщённых моделей, полностью подтвердило свою высокую эффективность в решении ряда нелинейных задач деформирования.

Вопросам распараллеливания численных методов определения напряжённо-деформированного состояния конструкций, и прежде всего метода конечного элемента, посвящено значительное число работ. Обзоры таких работ можно найти в публикациях $[3,4]$. Наиболее перспективными оказались методы, основанные на концепции декомпозиции. Они обеспечивают эффективное решение систем линейных алгебраических уравнений, преобразованных в соответствии с разделением всей конструкции на подобласти или с разделением матриц на подматрицы без явной связи с физической декомпозицией. Обсчёт каждой из подобластей (подматриц) выполняется независимо, что создает необходимые предпосылки для параллельных вычислений.

Метод декомпозиции был распространен и на анализ нелинейных задач деформирования. Рассмотрены физически и геометрически нелинейные задачи, ползучесть, оптимизация формы, связанные задачи и др. [4]. Методы, которые были использованы для введения параллельных вычислений, пред-

Яков Мордухович Клебанов (д.ф.-м.н., проф.), зав. кафедрой, каф. механики. Игорь Евгенъевич Адеянов (к.т.н.), ст. преподаватель, каф. механики. 
полагают итерационное или пошаговое решение исходной нелинейной задачи. На каждой итерации или каждом шаге в параллель решается линеаризованная задача. Для этого используется какой-либо известный метод параллельного решения линейных задач. Поэтому алгоритмы решения нелинейных задач являются модификацией соответствующих линейных алгоритмов и практически воспроизводят тот же метод декомпозиции, что и в линейной версии. При этом операции приведения параметров подобластей, занимающие значительный объём машинного времени, и обмен информацией между процессорами повторяются на всех многочисленных итерациях или шагах решения. В результате при таком подходе к распараллеливанию эффективность решения резко снижается.

В данной работе применяется альтернативный подход, свободный от указанных недостатков. Он распространяется на решение связанных задач пластического разупрочнения и повреждённости.

За последние годы получены важные теоретические результаты в области создания определяющих уравнений пластичности материалов с разупрочнением. Модели нелокальной пластичности интегрального вида считаются более обоснованными как с физической точки зрения, так и с позиции численной реализации [5]. В этих моделях деформации, традиционно определяемые как градиент перемещений, являются локальными. Нелокальные деформации определяются как интеграл от произведения локальной деформации и весовой функции, взятый по объёму тела в окрестности рассматриваемой точки. Таким образом, на величину нелокальной деформации в данной точке влияют локальные деформации в её окрестности. Это влияние тем меньше, чем больше расстояние между точками. Количественно влияние зависит от величины, называемой линейным размером характерного объёмного элемента. Данный размер связан с микроструктурой и механизмом разрушения при пластическом сдвиге, с деформацией зёрен, внутризёренным разрушением, движением дислокаций, образованием, ростом и слиянием вакансий и т. д. Характерный объёмный элемент линейного размера $l$ должен быть достаточно велик для того, чтобы включать в себя необходимое число микропустот и микротрещин, но в то же время он должен быть настолько мал, чтобы напряженное и деформированное состояние макротела можно было рассматривать как однородное или с небольшими неоднородностями. Минимальный размер характерного объёмного элемента зависит от микроструктурной неоднородности рассматриваемого материала. В работе [6] были предложены следующие характерные величины: металлы и керамика - $0,1 \mathrm{~mm}^{3}$; полимеры и композиты - $1 \mathrm{mм}^{3}$; дерево - $10 \mathrm{~mm}^{3}$; бетон - $100 \mathrm{mм}^{3}$. В качестве нелокальных деформаций обычно рассматриваются скалярные величины, определяемые по интенсивности локальных деформаций. Примеры экспериментальной проверки таких моделей и определения параметров внутренних шкал для расчета связанных процессов пластического деформирования и повреждаемости приводятся в работах $[7,8]$. На основе моделей нелокальной пластичности интегрального вида были разработаны градиентные методы, обобщающие локальную теорию пластичности путем включения в уравнения производных перемещений высокого порядка.

Решение краевых задач нелокальной пластичности выполняется с помощью коммерческих [9] или специально разработанных программных пакетов 
метода конечного элемента [10] и метода граничного элемента [11]. В большинстве работ предполагается полная связанность процессов деформирования и повреждаемости на всех этапах расчета. Имеются также исследования, применяющие методы неполной связанности.

Подход с разделением операторов существенно облегчает введение нелокальных величин - для их определения можно создавать соответствующие процедуры в существующих коммерческих профессиональных пакетах MKЭ. Данная статья посвящена подробному изложению одного из вариантов такого подхода, получившему название метода эквивалентной неповреждённой среды.

Основные уравнения. С помощью принципа эквивалентности деформаций реальной деформируемой среде ставится в соответствие некоторая эквивалентная среда без повреждённости. Приведённое напряжение $s_{i j}$ связывается с обычным напряжением $\sigma_{i j}$ зависимостью

$$
s_{i j}=\frac{\sigma_{i j}}{1-\omega}
$$

где $\sigma_{i j}$ - тензор напряжений; $s_{i j}$ - тензор приведённых напряжений; $\omega$ - скалярный параметр повреждаемости, $\omega \in[0,1)$. С учётом (1) уравнения равновесия

$$
\sigma_{i j, i}-X_{j}=0
$$

где $X_{j}$ - объёмные силы, могут быть преобразованы к виду

$$
s_{i j, i}-Y_{j}=0 .
$$

Здесь $Y_{j}$ - приведённые объёмные силы, имеющие вид

$$
Y_{j}=\frac{1}{1-\omega} s_{i j} \omega,{ }_{i}+\frac{X_{j}}{1-\omega} .
$$

Уравнение (3) является уравнением равновесия для эквивалентной среды без повреждаемости. Здесь и далее в работе, если не оговорено другое, индексы в тензорной записи принимают значения $1,2,3$.

Для регуляризации задачи пластического разупрочнения вводится нелокальная пластическая деформация $\bar{\varepsilon}_{p}$. Зависимость между нелокальной и локальной величинами записывается в неявной градиентной форме

$$
\bar{\varepsilon}_{p}-l^{2} \bar{\varepsilon}_{p}, i i=\varepsilon_{p} .
$$

Полученная система определяющих уравнений задачи прочности градиентной упругопластичности кроме уравнений (3)-(5) включает следующие зависимости:

$$
\begin{gathered}
\varepsilon_{i j}=0,5\left(u_{i, j}+u_{j}, i\right), \\
\varepsilon_{i j}=\frac{\partial \Phi\left(s_{k l}\right)}{\partial s_{i j}}, \\
\bar{\varepsilon}_{p, i} n_{i}=0 \text { на } \quad \Gamma, \\
\omega=\omega\left(\bar{\varepsilon}_{p}\right),
\end{gathered}
$$




$$
\omega<\omega_{\mathrm{Kp}}<1
$$

где $u_{i}$ - вектор перемещений, $\Phi$ - дополнительная работа приведённых напряжений, $n_{i}$ - единичная внешняя нормаль к внешней границе Г области, охватываемой телом.

Уравнение (7)- общий вид зависимости между компонентами напряжений и деформаций градиентального типа; уравнение (8) - однородное граничное условие Неймана для уравнения (5). Соотношение (9) - феноменологическая зависимость параметра повреждаемости $\omega$ от величины нелокальной эффективной пластической деформации $\bar{\varepsilon}_{p}$. Условие прочности (10) содержит критическое значение повреждаемости $\omega_{\text {кр }}$, при котором материал считается разрушенным.

К эквивалентной среде может быть применен подход к распараллеливанию решений, введенный в $[1,2]$. Этот подход основан на использовании аппроксимирующих обобщённых моделей подконструкций. Конструкция рассматривается как составленная из подконструкций. Каждая подконструкция аппроксимируется конечноэлементной сеткой.

Рассмотрим конструкцию, занимающую объём $\Omega$, и разделим её на $N$ подконструкций с объёмами $\Omega_{\alpha}, \alpha=1,2, \ldots, N$. Пусть поведение материала подконструкции описывается уравнениями $(3),(6),(7)$ для условно неповреждённого материала.

По аналогии с [2] запишем для случая степенной зависимости между деформацией и приведённым напряжением определяющие уравнения в виде

$$
\varepsilon_{i j}=\frac{\partial \Phi_{\alpha}\left(x_{k}, s_{l m}\left(x_{k}\right)\right)}{\partial s_{i j}\left(x_{k}\right)}, \quad \Phi_{\alpha}\left(x_{k}, s_{i j}\right)=B_{\alpha}\left(x_{k}\right) \frac{s_{e \alpha}^{\nu_{\alpha}+1}}{\nu_{\alpha}+1}, \quad s_{e \alpha}^{2}=\Phi_{i j k l}^{(\alpha)} s_{i j} s_{k l},
$$

где $x_{k}$ - пространственные координаты точек конструкции; $s_{e \alpha}-$ эквивалентное напряжение; $B_{\alpha}-$ функция координат; $\Phi_{i j k l}^{(\alpha)}$ - тензор коэффициентов податливости анизотропного материала; $\nu_{\alpha}>1$ - константа для подконструкции с номером $\alpha$.

В соответствии с $[12,13]$ можно записать связь эквивалентной приведённой обобщённой силы и эквивалентного обобщённого перемещения в виде

$$
u_{\alpha}=B_{0 \alpha} Q_{\alpha}^{\nu_{\alpha}}
$$

где $B_{0 \alpha}$ - константа подконструкции; $u_{\alpha}$ - эквивалентное обобщённое перемещение подконструкции $\alpha ; Q_{\alpha}$ - эквивалентная приведённая обобщённая сила, действующая на подконструкцию $\alpha$.

Особенностью приведённых обобщённых сил является то, что их работа на обобщённых перемещениях равна работе приведённых напряжений на деформациях в подконструкции.

Линейной зависимости между обобщёнными перемещениями и обобщёнными силами $\left(\nu_{\alpha}=1\right)$ в пространстве обобщённых сил и в пространстве обобщённых перемещений соответствуют поверхности равной работы в форме гиперэллипсоидов. При $\nu_{\alpha}>1$ эти поверхности не являются гиперэллипсоидами. При трансформации поверхностей, вызванной изменением $\nu_{\alpha}$, имеются точки, сохраняющие свои координаты. Этим точкам соответствуют однородные напряжённо-деформированные состояния подконструкции и 
отвечающие им системы внешних приведённых сил или перемещений. Это обстоятельство можно легко проследить по ходу доказательств теорем о вложенных поверхностях диссипации [14].

Как было отмечено выше, поверхность постоянной диссипации при $\nu_{\alpha}>1$ не является гиперэллипсоидом. Однако любая существенная ее часть может быть с высокой степенью точности представлена как часть гиперэллипсоида. Если при одних и тех же внешних воздействиях на конструкцию поворот вектора сил или перемещений, действующих на подконструкцию, оказывается в пределах такого участка, то указанная аппроксимация может явиться основой создания эффективной обобщённой модели подконструкции.

Использование в качестве аппроксимирующей поверхности гиперэллипсоида объясняется стремлением получить векторно-линейную связь между приведёнными силами и перемещениями. Обобщённое перемещение в соответствии с такой аппроксимацией может быть представлено в виде

$$
u_{\alpha}=\left(c_{r s \alpha} u_{r} u_{s}\right)^{1 / 2}, \quad r, s=1,2, \ldots,
$$

где коэффициенты $c_{r s \alpha}$ являются функционалами поля деформаций, соответствующими точке аппроксимации подконструкции $\alpha$. Количество обобщённых перемещений $u_{r}$ равно числу степеней свободы подконструкции без учёта её перемещений как жёсткого целого.

Таким образом, аппроксимирующее обобщённое определяющее уравнение записывается в виде

$$
Q_{s}=c_{r s \alpha} u_{\alpha}^{\mu_{\alpha}-1} u_{r}, \quad 0<\mu_{\alpha} \leqslant 1 .
$$

Особенность зависимости (12) состоит в том, что здесь обобщённая сила является приведённой, т.е. записана для тела с условно неповреждённым материалом.

В работе [15] метод аппроксимирующих обобщённых моделей распространен на случай произвольной диаграммы деформирования. Основное соотношение записано в виде

$$
s_{i j}=\frac{\partial \Psi\left(x_{k}, \varepsilon_{l m}\right)}{\partial \varepsilon_{i j}} .
$$

Эквивалентное обобщённое перемещение также может быть представлено в виде (11).

Таким образом, аппроксимирующее обобщённое определяющее уравнение может быть записано в виде

$$
Q_{s}=d_{r s \alpha} u_{r} \frac{1}{u_{\alpha}^{\prime}} \frac{\partial \tilde{\Psi}_{\alpha}}{\partial u_{\alpha}^{\prime}}
$$

где $d_{r s \alpha}=\lambda_{\alpha} c_{r s \alpha}, u_{\alpha}^{\prime}=\left(d_{r s \alpha} u_{r} u_{s}\right)^{1 / 2}, \tilde{\Psi}_{\alpha}\left(u_{\alpha}^{\prime}\right)=\Psi_{\alpha}\left(u_{s}\right)$. Здесь $\lambda_{\alpha}$ - скалярный параметр, величина которого корректируется на каждой итерации.

При степенной диаграмме неизвестными параметрами обобщённой зависимости $(11),(12)$ являются входящие в неё коэффициенты $c_{r s \alpha}$. При произвольной диаграмме деформирования материала неизвестной является также и форма обобщённой диаграммы. Поэтому в процессе итераций при решении 
каждой конкретной задачи не только определяются неизвестные константы, но и восстанавливается форма диаграммы.

Алгоритм параллельного решения задачи прочности при пластическом разупрочнении материала. Полученные выше зависимости позволяют сформировать итерационные алгоритмы параллельного решения краевых задач пластичности при разупрочнении.

Для случая эквивалентной среды с произвольной диаграммой деформирования разработан следующий алгоритм решения.

ЭтАП 0. Создаётся твёрдотельная модель. Задаются типы конечных элементов, их размер, тип сетки (свободная или регулярная), создаётся конечноэлементная модель конструкции. Задаются свойства материала - упругие константы и диаграмма «приведённые напряжения деформация». Задаются граничные условия.

ЭтАП 1. В качестве нулевого приближения методом суперэлементов для рассматриваемого тела при заданных внешних условиях решается краевая задача в предположении линейных свойств материала. Решение на стадиях приведения подконструкций и локального анализа для каждой подконструкции выполняется в параллель. На стадии решения глобальной задачи решение осуществляется последовательно. В качестве результата получаются перемещения $u_{s}$ узлов глобальной модели, то есть узлов на границах подконструкций. Определяется первая точка нелинейной части диаграммы « $Q_{\alpha}-u_{\alpha}$, отвечающая достижению в наиболее нагруженном конечном элементе предела текучести.

ЭТАП 2. По результатам предшествующих вычислений для каждой подконструкции формируются векторы заданных перемещений внешних узлов $u_{s}^{(p-1)}$, где $p$ - номер итерации. Проводится нелинейный локальный анализ подконструкций при заданных перемещениях $u_{s}^{(p-1)}$. Решение нелинейной задачи (3), (6), (7) выполняется для эквивалентной неповреждённой среды.

ЭТАП 3. Выполняется решение дополнительной линейной краевой задачи (7), (8) для всей конструкции методом суперэлементов и определяются повреждаемость и дополнительные приведённые объёмные силы в соответствии с (9) и (4) соответственно.

ЭТАП 4. Производится проверка сходимости в соответствии с условием

$$
\left|\omega_{k}^{\max }-\omega_{k-1}^{\max }\right|<\delta^{\prime}
$$

где $\omega_{k}^{\max }$ - максимальное значение повреждаемости в конечном элементе подконструкции на $k$-той итерации, $\delta^{\prime}$ - допустимая величина погрешности. Если данный критерий не выполняется, то происходит возврат к этапу 2.

ЭТАП 5. Находятся $c_{r s \alpha}^{(p-1)}$ и формируются аппроксимирующие обобщённые модели подконструкций в соответствии с (11). Параметр $\lambda$ определяется из условия нормирования

$$
\lambda c_{r s \alpha}^{(p-1)} u_{r}^{(p-1)} u_{s}^{(p-1)}=d_{r s \alpha}^{(p-2)} u_{r}^{(p-1)} u_{s}^{(p-1)} .
$$

Это условие обеспечивает непрерывность изменения эквивалентного обобщённого перемещения при изменении матриц $c_{r s \alpha}$. 
ЭТАП 6. Итерациями решается нелинейная глобальная задача для всей конструкции, составленной из подконструкций. Сходимость итерационного процесса оценивается по относительному изменению эквивалентных приведённых обобщённых сил во всех подконструкциях. Это изменение на очередной итерации не должно превышать заданной величины. Для каждой подконструкции используется своя диаграмма «эквивалентная приведённая обобщённая сила - эквивалентное перемещение». Результатом являются перемещения $u_{s}^{p}$ во внешних узлах.

ЭтАП 7. Оценивается достигнутая точность решения глобальной задачи:

$$
\sqrt{\sum_{s}\left(u_{s}^{m}-u_{s}^{m-1}\right)^{2} / \sum_{s}\left(u_{s}^{m}\right)^{2}}<\delta^{\prime \prime},
$$

где $s$ - номер внешнего узла, $m$ - номер внешней итерации, $\delta^{\prime \prime}$ - допустимая величина погрешности. При необходимости повторяются этапы 2-7.

ЭТАП 8. Выполняется локальный анализ подконструкций, проверяется выполнение условия прочности (10). В качестве исходных данных здесь выступают полученные при глобальном анализе (этап 6) перемещения внешних узлов.

Из приведённого алгоритма видно, что одна итерация включает этапы $2-7$. Нелинейная задача на этапе 2 может решаться любым известным методом решения задач деформационной теории пластичности. На каждой итерации осуществляется последовательная аппроксимация диаграммы « $Q_{\alpha}-u_{\alpha} »$ каждой подконструкции. Соседние точки диаграмм соединяются прямыми линиями. Также итерациями решается нелинейная глобальная задача для всей конструкции в главной сети на этапе 6 . Анализ каждой подконструкции на этапах 2, 5, 8 выполняется без какого-либо обмена информацией между подконструкциями. Обычно предполагается, что каждый процессор работает со своей индивидуальной подконструкцией, и, следовательно, на этих этапах могут быть достигнуты идеальное ускорение и наивысшая эффективность распараллеливания.

Численная реализация. Предложенный подход был реализован с помощью метода конечных элементов. Была разработана программа, использующая представленный выше алгоритм. Ниже рассмотрено решение тестовой задачи растяжения пластинки с острой несимметричной выточкой, иллюстрирующей особенности поведения конструкций в условиях разупрочнения.

Конечноэлементная модель пластинки представлена на рис. 1. Она разбита на две подконструкции, граница между которыми выделена жирной линией. В меньшей по объёму подконструкции, включающей выточку, для повышения точности расчёта взята более мелкая сетка конечных элементов.

Диаграмма «приведённые напряжения - деформация» представлена на рис. 2. Зависимость изменения параметра повреждаемости от нелокальной пластической деформации принята в линейном виде

$$
\omega=\bar{\varepsilon}_{p} / \bar{\varepsilon}_{p}^{0}, \quad \omega<\omega_{\text {кр }}=0,57,
$$




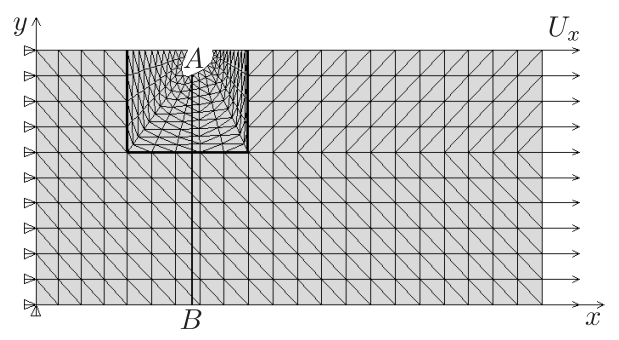

Рис. 1. Конечно-элементная модель и условия нагружения плоской пластинки с выточкой

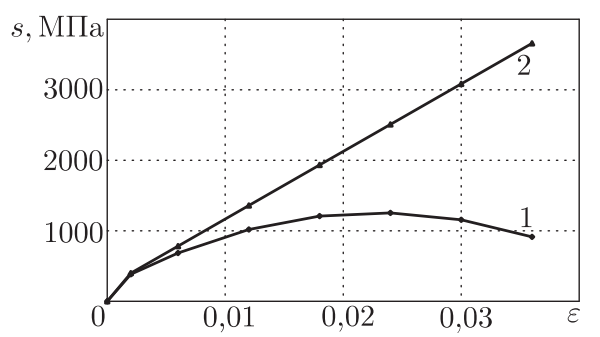

Рис. 2. Диаграмма «напряжения - деформации» для материала полосы с односторонней выточкой: 1 - истинная; 2 - приведённая

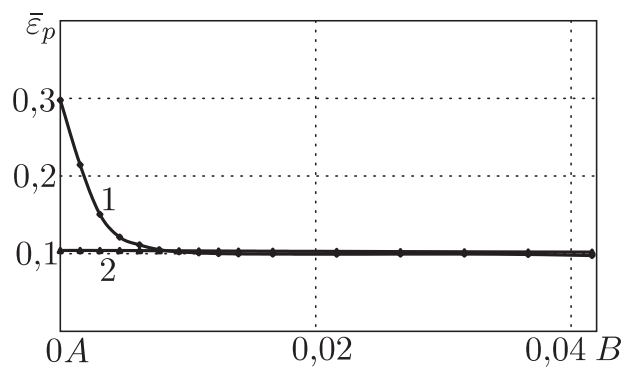

Рис. 3. Распределение интенсивности пластической деформации $\bar{\varepsilon}_{p}$ по сечению $A-B$ пластинки: 1 -интенсивность локальной пластической деформации; 2 - интенсивность нелокальной пластической деформации

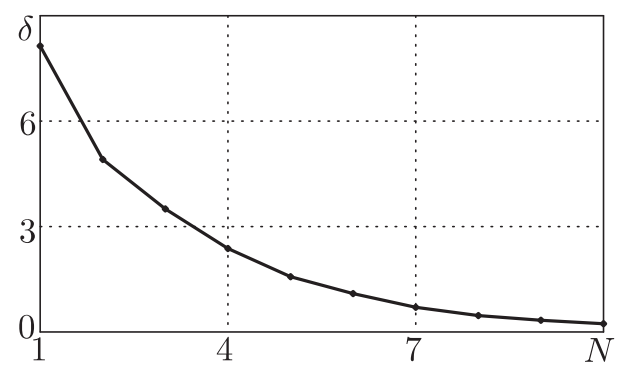

Рис. 4. Зависимость величины относительной погрешности $\delta(\%)$ от числа итераций $N$ где $\bar{\varepsilon}_{p}^{0}$ - константа. Нужно отметить, что для определения значений констант $\bar{\varepsilon}_{p}^{0}$ и $\omega_{\text {кр }}$, используемых для построения диаграммы «приведённые напряжения - деформация», в общем случае кроме стандартных испытаний образцов, в которых реализуется однородное напряжённо-деформированное состояние, требуется проведение испытаний, реализующих неоднородное состояние. Соответствующую методику можно найти, например, в работе [16].

Расчёты выполнялись в соответствии с алгоритмом, приведённым выше.

На рис. 3, 4 приведены результаты расчётов для двухмерной пластинки с выточкой при максимальном значении повреждаемости 0,56. Расчёты выполнялись для различных конечно-элементных сеток. Более мелкая сетка даёт качественно ту же картину, что и более крупная. Количественные различия незначительны. Из рис. 3 видно, что концентрация пластической деформации существенно выше, чем концентрация нелокальной пластической деформации. На рис. 4 показан процесс сходимости итерационной процедуры, погрешность которого оценивается в соответствии с условием (12). Важно то, что этот процесс является равномерным.

Анализ временных затрат и эффективности параллельной реализации. При решении задачи пластичности с разупрочнением на параллельной ЭВМ, когда число процессоров равно числу подконструкций, в соответствии с приведённым алгоритмом общее время складывается из трёх компонент [15]:

$$
T=T_{\mathrm{I}}+T_{\mathrm{II}}+T_{\mathrm{III}},
$$

где $T_{\mathrm{I}}$ - время вычислений, выполняемых с максимальным распарал- 
леливанием; $T_{\text {II }}$ - время вычислений, выполняемых последовательно на одном процессоре; $T_{\mathrm{III}}$ - время вспомогательных вычислений.

Структура временных затрат, образующих $T_{\mathrm{I}}$, в соответствии с разработанным алгоритмом имеет вид

$$
T_{\mathrm{I}}=\max _{\alpha}\left(t_{1}^{\alpha}+t_{2}^{\alpha}\right)+\sum_{p=1}^{k_{1}}\left\{\max _{\alpha}\left[k_{2 p}^{\alpha}\left(t_{1}^{\alpha}+t_{3}^{\alpha}\right)\right]\right\}+\sum_{p=1}^{k_{1}}\left\{\max _{\alpha}\left[k_{3 p}^{\alpha}\left(t_{1}^{\alpha}+t_{3}^{\alpha}\right)\right]\right\},
$$

где индекс $\alpha$ соответствует номеру подконструкции, а индекс $p$ - номеру итерации, включающей этапы $2-7 ; k_{1}$ - число таких итераций; $k_{2 p}^{\alpha}$ - число итераций при приведении подконструкции $\alpha$ (этап 2 ) на итерации $p ; k_{3 p}$ - число итераций при решении нелинейной задачи в главной сети (этап 6) на итерации $p$, зависящее от условий деформирования, показателя степени нелинейности $\nu_{\alpha}$ и задаваемой точности; $t_{1}^{\alpha}, t_{2}^{\alpha}$ и $t_{3}^{\alpha}$ - времена основных операций.

В формулу (13) входят только времена наиболее длительных операций, каковыми являются: $t_{1}^{\alpha}$ - построение матрицы жёсткости подконструкции, $t_{2}^{\alpha}$ - «конденсация» матрицы жёсткости подконструкции к внешним степеням свободы и $t_{3}^{\alpha}$ - время решения системы линейных уравнений в подконструкции. Время, затрачиваемое на все остальные операции, значительно меньше указанных времён, и поэтому оно отнесено к $T_{\mathrm{III}}$.

Основными факторами, определяющими время вычислений $T_{\mathrm{I}}$, являются число элементов и число внутренних степеней свободы в подконструкциях, общее число степеней свободы и полуширина ленты матрицы жёсткости, количество итераций при приведении подконструкций $k_{2 p}^{\alpha}$ и $k_{1}$. Количество итераций, в свою очередь, зависит от напряжённо-деформированного состояния подконструкции, степени нелинейности определяющих уравнений $\nu_{\alpha}$ и задаваемой точности решения.

Рассмотрим теперь основные факторы, определяющие время последовательных вычислений в главной сети, выполняемых на одном процессоре

$$
T_{\mathrm{II}}=t_{4}+t_{5}+\sum_{p=1}^{k_{1}} k_{2 p} t_{5}+\sum_{p=1}^{k_{1}}\left[k_{3 p}\left(t_{4}+t_{5}\right)\right]
$$

где $t_{4}$ - время построения матрицы жёсткости главной сети; $t_{5}$ - время решения системы линейных уравнений в главной сети. Первые два слагаемых в (14) соответствуют этапу 1 алгоритма решения задачи. Второе слагаемое определяет время последовательных вычислений на этапе 3. В третьем слагаемом представлено время, необходимое для последовательных вычислений на этапе 6 .

Основными факторами, определяющими время вычислений $T_{\mathrm{II}}$, являются число подконструкций, число степеней свободы в главной сети, полуширина ленты матрицы жёсткости, а также число итераций при решении нелинейной задачи в главной сети $k_{3 p}$ и число больших нелинейных итераций $k_{1}$.

Время вспомогательных вычислений $T_{\mathrm{II}}$ включает в себя время операций ввода-вывода, время запуска необходимого числа процессоров, время обмена данными между процессорами, время инициализации массивов данных и время для прочих вспомогательных вычислений. Это время составляет от 1 до $10 \%$ от общего времени решения задачи в зависимости от размера решаемой 
задачи, числа используемых процессоров, числа и размера подконструкций и т. д. Необходимо отметить, что время $T_{\mathrm{III}}$ становится менее значимым при увеличении размера решаемой задачи, так как оно прямо пропорционально размеру задачи, в то время как время операций «конденсации» матрицы жёсткости или решения системы линейных уравнений пропорционально квадрату или кубу величин, зависящих от размера задачи.

Проанализировав структуру времён $T_{\mathrm{I}}, T_{\mathrm{II}}, T_{\mathrm{II}}$, можно сделать вывод, что при неизменном общем размере задачи с увеличением числа подконструкций уменьшается их размер и соответственно время вычислений в каждой подконструкции $T_{\mathrm{I}}$, но одновременно увеличивается время вычислений в главной сети $T_{\text {II }}$. Следовательно, время решения задачи зависит от соотношения размеров задач для подконструкций и главной сети. Поэтому существует оптимальное соотношение, при котором общее время решения является минимальным. Используя формулы (14)-(16), ещё до проведения расчётов можно дать рекомендации по декомпозиции элемента конструкции на подконструкции с тем, чтобы уменьшить время решения.

Упомянутая выше задача растяжения пластинки, разбитой на две подконструкции, решалась параллельным алгоритмом на одно- и двухпроцессорной ӘВМ. Ниже приводятся значения времени, затраченного на решение задачи, сек:

время расчёта первой подконструкции на одном процессоре . . . 4,282

время расчёта второй подконструкции на одном процессоре . . . 3,452

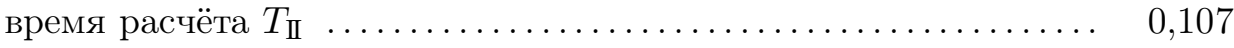

время расчёта $T_{\mathrm{I}}$ при расчёте на двух процессорах $\ldots \ldots \ldots \ldots \ldots \quad 4,282$

суммарное время расчёта задачи на двух процессорах ....... 4, 4,389

суммарное время расчёта задачи на одном процессоре . . . . . . 6. 6,672

время расчёта без подконструкций на одном процессоре . . . . . 6 6,200

Важными характеристиками параллельного алгоритма являются ускорение $S_{p}$ и эффективность $E_{p}$ :

$$
S_{p}=T_{1} / T_{p}, \quad E_{p}=S_{p} / p \cdot 100 \%,
$$

где $T_{1}$ - время выполнения параллельного алгоритма на одном процессоре, $T_{p}$ - время выполнения параллельного алгоритма в системе из $p$ процессоров. При расчёте пластинки на двухпроцессорной ӘВМ было получено ускорение $S_{p}=1,52$, что соответствует эффективности распараллеливания $E_{p}=76 \%$. Абсолютное значение выигрыша по времени в данной задаче незначительно в силу малой размерности. Но для задач с большим числом степеней свободы и количеством подконструкций распараллеливание позволит получить существенный выигрыш и в абсолютном значении затрат машинного времени.

Выводы. Таким образом, на основе предложенной авторами методики решения задач прочности конструкций при пластическом разупрочнении материала были разработаны алгоритмы решения соответствующих задач, опирающиеся на использование концепции эквивалентной неповреждённой среды. Представлен наиболее общий из этих алгоритмов. В нём реализуется итерационная процедура, позволяющая осуществить численную реализацию этого алгоритма на практике с распараллеливанием процесса решения. С помощью программы, реализующей данный алгоритм, выполнен расчёт пластинки с выточкой, показавший хорошую эффективность распараллеливания. 


\section{БИБЛИОГРАФИЧЕСКИЙ СПИСОК}

1. Klebanov I.M., Davydov A.N. A non-linear domain decomposition method: http://ansys.net/collection/746.

2. Klebanov I. M., Davydov A.N. A parallel computational method in steady power-law creep // Int. J. Num. Methods Eng., 2001. Vol. 50, no. 8. Pp. 1825-1840.

3. Yagawa G., Yoshioka A., Soneda S. A parallel finite element method with a supercomputer network // Comput. Struct., 1993. Vol. 47, no. 3. Pp. 407-418.

4. Asta M., Fischera R., Labartab J., Manza H. Run-time parallelization of large FEM analyses with PERMAS // Adv. Eng. Soft., 1998. Vol. 29, no. 3-6. Pp. 241-248.

5. Aifantis E. C. On the role of gradients in the localization of deformation and fracture // Int. J. Eng. Sci., 1992. Vol. 30, no. 10. Pp. 1279-1300.

6. Lemaître J. A course on Damage Mechanics. Berlin: Springer, 1996. 228 pp.

7. Shu J. Y., Barlow C. Y. Strain gradient effects on microscopic strain field in a metal matrix composite// Int. J. Plast., 2000. Vol. 16, no. 5. Pp. 563-591.

8. Geers M. G.D., de Borst R., Peerlings R.H.J. Validation and internal length scale determination for a gradient damage model: application to short glass-fibre-reinforced polypropylene// Int. J. Solids Struct., 1999. Vol.36, no. 17. Pp. 2557-2584.

9. Nygårds M., Gudmundson P. Numerical investigation of the effect of non-local plasticity on surface roughening in metals // Eur. J. Mech., A, Solids, 2004. Vol. 23, no. 5. Pp. 753-762.

10. Baaser H., Tvergaard $V$. A new algorithmic approach treating nonlocal effects at finite rate-independent deformation using the Rousselier damage model // Comput. Methods Appl. Mech. Eng., 2003. Vol. 192, no. 1-2. Pp. 107-124.

11. Botta A.S., Venturini W.S., Benallal A. BEM applied to damage models emphasizing localization and associated regularization techniques // Eng. Anal. Bound. Elem., 2005. Vol. 29, no. 8. Pp. 814-827.

12. Качанов Л. М. Теория ползучести. М.: Физматгиз, 1960. 390 с. [Kachanov L.M. Theory of creep. Moscow: Fizmatgiz, 1960. 390 pp.]

13. Boyle J. T., Spence J. Stress analysis for creep. London: Butterworth, 1983. 284 pp.

14. Клебанов Я. М., Самарин Ю. П. Вложенные поверхности мощности диссипации в пространстве сил и скоростей перемещений при установившейся ползучести неоднородных и анизотропных тел // Механика твердого тела, 1997. №6. С. 121-125. [Klebanov Ya. M., Samarin Yu.P. Nested power dissipation surface in forces and displacements space for the steady creep of heterogeneous and anisotropic bodies // Mekhanika Tverdogo Tela, 1997. no. 6. Pp. 121-125].

15. Клебанов Я. М., Давыдов А. Н. Параллелизация нелинейных задач при произвольной диаграмме деформирования // Вестн. Сам. гос. техн. ун-та. Сер. Техн. науки, 2000. №10. C. 21-25. [Klebanov Ya.M., Davydov A. N. Parallel solution of nonlinear problems with an arbitrary deformation diagram// Vestn. Samar. Gos. Tekhn. Un-ta. Ser. Tekhn. Nauki, 2000. no. 10. Pp. 21-25].

16. de Borst R., Pamin J., Geers M. G.D. On coupled gradient-dependent plasticity and damage theories with a view to localization analysis // Eur. J. Mech., A, Solids, 1999. Vol. 18, no. 6. Pp. 939-962. 
MSC: 74S05; 74C99, 65Y05

\section{SOLUTION PARALLELIZATION OF SOFTENING PLASTICITY PROBLEMS}

\section{M. Klebanov, I. E. Adeyanov}

Самарский государственный технический университет, 443100, Самара, ул. Молодогвардейская, 244.

E-mails: jklebanov@mail.ru, adigorev@gmail.com

Parallelization of deformation-damage coupling boundary value problem solution is considered. In definition the equations of the strength we used the principle of equivalence of deformations in a real and relatively undamaged structures. Principle of real and hypothetical undamaged structure strain equivalence is applied. An iterative procedure characterized by successive solutions of plasticity and damage problems at each iteration step, is proposed. The approach to parallelization of softening plasticity boundary value problem solution is based on the conception of generalized nonlinear structural models and on the method of decomposition.

Key words: parallelization, structure, generalized model, decomposition, softening plasticity, boundary value problem, nonlocal plastic strain.

Original article submitted 20/XII/2010; revision submitted $16 / \mathrm{V} / 2011$.

Iakov M. Klebanov (Dr. Sci. (Phys. \& Math.)), Head of Dept., Dept. of Mechanics. Igor' E. Adeyanov (Ph. D. (Techn.)), Senior Teacher, Dept. of Mechanics. 